\title{
LOCALLY CONVEX SPACES AND SCHUR TYPE PROPERTIES
}

\author{
Saak Gabriyelyan \\ Ben-Gurion University of the Negev, Department of Mathematics \\ Beer-Sheva, P.O. 653, Israel; saak@math.bgu.ac.il
}

\begin{abstract}
In the main result of the paper we extend Rosenthal's characterization of Banach spaces with the Schur property by showing that for a quasi-complete locally convex space $E$ whose separable bounded sets are metrizable the following conditions are equivalent: (1) $E$ has the Schur property, (2) $E$ and $E_{w}$ have the same sequentially compact sets, where $E_{w}$ is the space $E$ with the weak topology, (3) $E$ and $E_{w}$ have the same compact sets, (4) $E$ and $E_{w}$ have the same countably compact sets, (5) $E$ and $E_{w}$ have the same pseudocompact sets, (6) $E$ and $E_{w}$ have the same functionally bounded sets, (7) every bounded non-precompact sequence in $E$ has a subsequence which is equivalent to the unit basis of $\ell_{1}$ and (8) every bounded non-precompact sequence in $E$ has a subsequence which is discrete and $C$-embedded in $E_{w}$.
\end{abstract}

\section{Introduction}

A locally convex space (lcs for short) $E$ is said to have the Schur property if $E$ and $E_{w}$ have the same convergent sequences, where $E_{w}$ is the space $E$ endowed with the weak topology $\sigma\left(E, E^{\prime}\right)$. By the classical Schur theorem the Banach space $\ell_{1}(\Gamma)$ has the Schur property for every set $\Gamma$. The results of Rosenthal in [38] (see also $\S 2$ of [8]) show that a Banach space $E$ has the Schur property if and only if every $\delta$-separated sequence in the closed unit ball of $E$ has a subsequence equivalent to the unit basis of $\ell_{1}$. Recall that a sequence $\left\{x_{n}\right\}_{n \in \mathbf{N}}$ is said to be $\delta$-separated if $\left\|x_{i}-x_{j}\right\| \geq \delta$ for all distinct $i, j \in \mathbf{N}$. Since any bounded sequence in $E$ either is precompact or contains a $\delta$-separated subsequence for some $\delta>0$, following [26] the above characterization of Banach spaces with the Schur property can be formulated in a more convenient topological form.

Theorem 1.1. (Rosenthal) A Banach space $E$ has the Schur property if and only if every bounded non-precompact sequence in $E$ has a subsequence which is equivalent to the unit basis of $\ell_{1}$.

Another characterization of Banach spaces with the Schur property was obtained recently by Dowling, Freeman, Lennard, Odell, Randrianantoanina and Turett in [15]: A Banach space $E$ has the Schur property if and only if every weakly compact subset of $E$ is contained in the closed convex hull of a weakly null sequence. A short proof of this result is given by Johnson, Lillemets and Oja in [31]. For other characterizations of Banach spaces with the Schur property see [7, 26, 36].

It is well known that a Banach space $E$ has the Schur property if and only if $E$ and $E_{w}$ have the same compact sets, and therefore, by the Eberlein-Šmulyan theorem, if and only if $E$ and $E_{w}$ have the same sequentially compact sets if and only if $E$ and $E_{w}$ have the same countably compact sets if and only if $E$ and $E_{w}$ have

https://doi.org/10.5186/aasfm.2019.4417

2010 Mathematics Subject Classification: Primary 46A03, 46E10.

Key words: Schur property, weak respecting property, Dunford-Pettis property, sequential Dunford-Pettis property. 
the same pseudocompact sets (the last equivalence follows from a result of Pták, see $[32, \S 24.3(7)])$. An analogous statement holds also for strict $(L F)$-spaces, see [32, $\S 24.3(9)$ ]. Moreover, a remarkable result of Valdivia [40] (which states that $E_{w}$ is a $\mu$-space for every quasi-complete space $E$ ) easily implies the following assertion: If $E$ and $E_{w}$ have the same compact sets, then $E$ and $E_{w}$ have the same functionally bounded subsets. Note that in this assertion one cannot replace compact sets by convergent sequences even for complete spaces, see Proposition 3.5 below.

For a Tychonoff (= completely regular Hausdorff) space $X$ and a property $\mathcal{P}$, denote by $\mathcal{P}(X)$ the set of all subspaces of $X$ with $\mathcal{P}$. Analogously to the corresponding algebraic notion we shall say that a locally convex space $E$ weakly respects $\mathcal{P}$ if $\mathcal{P}\left(E_{w}\right)=\mathcal{P}(E)$. So every lcs $E$ weakly respects bounded sets, and the Schur property means that $E$ weakly respects the property $\mathcal{S}$ of being a convergent sequence. The following theorem extends Theorem 1.1 and is the main result of the paper (recall that an lcs $E$ is said to be quasi-complete if each closed bounded subset of $E$ is complete).

Theorem 1.2. Let $E$ be a quasi-complete lcs whose separable bounded sets are metrizable. Then the following assertions are equivalent:

(i) E has the Schur property;

(ii) E weakly respects sequential compactness;

(iii) $E$ weakly respects compactness;

(iv) $E$ weakly respects countable compactness;

(v) E weakly respects pseudocompactness;

(vi) $E$ weakly respects functional boundedness;

(vii) every bounded non-precompact sequence in $E$ has a subsequence which is equivalent to the unit basis of $\ell_{1}$;

(viii) every bounded non-precompact sequence in $E$ has a subsequence which is discrete and $C$-embedded in $E_{w}$.

If (i)-(viii) hold, then every functionally bounded subset in $E_{w}$ is relatively compact in $E$.

Note that for any lcs E, (i) and (ii) are equivalent and conditions (iii)-(v) imply the Schur property, see Proposition 2.2. However, in general the Schur property does not imply the weak respecting compactness (see [42, Example 6 (p. 267)] and [14, Example 19.19] or the more general Proposition 3.5 below), and hence the condition of being metrizable for separable bounded sets in Theorem 1.2 is essential. We prove Theorem 1.2 in Section 2 using (1) an extension of the Rosenthal $\ell_{1}$ theorem obtained recently by Ruess [39], and (2) the aforementioned result of Valdivia [40]. As a corollary of Theorem 1.2 we provide numerous characterizations of the Schur property for strict $(L F)$-spaces, see Corollary 2.13.

It is well known that every Banach space with the Schur property has the Dunford-Pettis property. Being motivated by this result and the results of Albanese, Bonet and Ricker [1] and Bonet and Lindström [5], in the last section we study convergent sequences in duals of locally convex spaces with the Schur property and Dunford-Pettis type properties and extend some results known for Banach spaces and Fréchet spaces.

\section{Proof of Theorem 1.2}

A subset $A$ of a Tychonoff space $X$ is called functionally bounded in $X$ if every continuous real-valued function on $X$ is bounded on $A$, and $X$ is a $\mu$-space if every 
functionally bounded subset of $X$ has compact closure. Following [21], a topological space $X$ is a countably $\mu$-space if every countable functionally bounded subset of $X$ has compact closure. Clearly, every $\mu$-space is a countably $\mu$-space, but the converse is not true in general.

In what follows we consider the following families of compact type properties

$$
\mathfrak{P}_{0}:=\{\mathcal{S}, \mathcal{C}, \mathcal{S C}, \mathcal{C C}, \mathcal{P C}\} \quad \text { and } \quad \mathfrak{P}:=\mathfrak{P}_{0} \cup\{\mathcal{F} \mathcal{B}\},
$$

where $\mathcal{S}, \mathcal{C}, \mathcal{S C}, \mathcal{C C}, \mathcal{P C}$ or $\mathcal{F B}$ denote the property of being a convergent sequence or being a compact, sequentially compact, countably compact, pseudocompact and functionally bounded subset of a topological space, respectively.

Proposition 2.1. Let $H$ be a subspace of an lcs $E$ and $\mathcal{P} \in \mathfrak{P}_{0}$. If $E$ weakly respects $\mathcal{P}$, then $H$ weakly respects $\mathcal{P}$ as well.

Proof. Let $K \in \mathcal{P}\left(H_{w}\right)$. Denote by $i: H \rightarrow E$ the identity map. Then $i$ is weakly continuous. So $K=i(K) \in \mathcal{P}\left(E_{w}\right)$ (note that if $K$ is pseudocompact, then $i(K)$ is also pseudocompact by $[17,3.10 .24])$. Hence $K \in \mathcal{P}(E)$. Therefore $K \in \mathcal{P}(H)$. Thus $H$ weakly respects $\mathcal{P}$.

It is clear that if an lcs $E$ weakly respect compactness then it has the Schur property. Next proposition shows that the Schur property is weaker than other properties from $\mathfrak{P}$.

Proposition 2.2. Let $(E, \tau)$ be a locally convex space. Then:

(i) E has the Schur property if and only if it weakly respects sequential compactness;

(ii) if $E$ weakly respects countable compactness, then $E$ has the Schur property;

(iii) if $E$ weakly respects pseudocompactness, then $E$ has the Schur property;

(iv) if $E$ is a countably $\mu$-space and weakly respects functional boundedness, then $E$ has the Schur property;

(v) if every functionally bounded subset of $E_{w}$ has compact closure in $E$, then $E$ weakly respects all properties $\mathcal{P} \in \mathfrak{P}$ and $E_{w}$ is a $\mu$-space.

Proof. (i) Assume that $(E, \tau)$ has the Schur property and let $A$ be a sequentially compact subset of $E_{w}$. Take a sequence $S=\left\{a_{n}\right\}_{n \in \mathbf{N}}$ in $A$. Then $S$ has a weakly convergent subsequence $S^{\prime}$. By the Schur property $S^{\prime}$ converges in $\tau$. Hence $A$ is $\tau$-sequentially compact. Thus $E$ weakly respects sequential compactness.

Conversely, assume that $(E, \tau)$ weakly respects sequential compactness and let $\left\{a_{n}\right\}_{n \in \mathbf{N}}$ be a sequence weakly converging to an element $a_{0} \in E$. Set $S:=\left\{a_{n}\right\}_{n \in \mathbf{N}} \cup$ $\left\{a_{0}\right\}$, so $S$ is weakly compact. Being countable $S$ is metrizable and hence weakly sequentially compact. So $S$ is sequentially compact in $\tau$. We show that $a_{n} \rightarrow a_{0}$ in $\tau$. Suppose for a contradiction that there is a $\tau$-neighborhood $U$ of $a_{0}$ which does not contain an infinite subsequence $S^{\prime}$ of $S$. Then there is a subsequence $\left\{a_{n_{k}}\right\}_{k \in \mathbf{N}}$ of $S^{\prime}$ which $\tau$-converges to an element $g \in S$. Clearly, $g \neq a_{0}$ and $a_{n_{k}} \rightarrow g$ in the weak topology, and hence $a_{n} \not \rightarrow a_{0}$ in $\sigma\left(E, E^{\prime}\right)$, a contradiction. Therefore $a_{n} \rightarrow a_{0}$ in $\tau$. Thus $E$ has the Schur property.

(ii),(iii) Let $\left\{a_{n}\right\}_{n \in \mathbf{N}}$ be a sequence weakly converging to an element $a_{0} \in E$. Set $S:=\left\{a_{n}\right\}_{n \in \mathbf{N}} \cup\left\{a_{0}\right\}$, so $S$ is weakly compact. Hence $S$ is $\sigma\left(E, E^{\prime}\right)$-countably compact. So $S$ is countably compact or pseudocompact in $\tau$, respectively. As any countable space is normal, in both cases $S$ is countably compact in $\tau$. We show that $a_{n} \rightarrow a_{0}$ in $\tau$. Suppose for a contradiction that there is a $\tau$-neighborhood $U$ of $a_{0}$ which does not contain an infinite subsequence $S^{\prime}$ of $S$. Then $S^{\prime}$ has a $\tau$-cluster 
point $g \in S$ and clearly $g \neq a_{0}$. Note that $g$ is also a cluster point of $S^{\prime}$ in the weak topology. Hence $g=a_{0}$, a contradiction. Therefore $a_{n} \rightarrow a_{0}$ in $\tau$. Thus $E$ has the Schur property.

(iv) Let $S=\left\{a_{n}: n \in \mathbf{N}\right\} \cup\left\{a_{0}\right\}$ be a sequence in $E$ which weakly converges to $a_{0}$. Since $S$ is also functionally bounded in $E_{w}$, we obtain that $S$ is closed and functionally bounded in $E$. So $S$ is compact in $E$ because $E$ is a countably $\mu$-space. As the identity map $\left(S,\left.\tau\right|_{S}\right) \rightarrow\left(S,\left.\sigma\left(E, E^{\prime}\right)\right|_{S}\right)$ is a homeomorphism, $a_{n} \rightarrow a_{0}$ in $E$. Thus $E$ has the Schur property.

(v) Let $A \in \mathcal{P}\left(E_{w}\right)$. Then $A$ is functionally bounded in $E_{w}$. Therefore its $\tau$ closure $\bar{A}$ is compact in $E$, so the identity map id: $\left(\bar{A},\left.\tau\right|_{\bar{A}}\right) \rightarrow\left(\bar{A},\left.\sigma\left(E, E^{\prime}\right)\right|_{\bar{A}}\right)$ is a homeomorphism. Hence $E_{w}$ is a $\mu$-space and $A \in \mathcal{P}(E)$. Thus $E$ weakly respects $\mathcal{P}$.

Recall that a locally convex space $E$ is called semi-Montel if every bounded subset of $E$ is relatively compact, and $E$ is a Montel space if it is a barrelled semi-Montel space.

Proposition 2.3. A semi-Montel space $E$ weakly respects all properties $\mathcal{P} \in \mathfrak{P}$.

Proof. Let $A \in \mathcal{P}\left(E_{w}\right)$. Then $A$ is a functionally bounded subset of $E_{w}$ and hence $A$ is bounded in $E$. Therefore the closure $\bar{A}$ of $A$ in $E$ is compact and (v) of Proposition 2.2 applies.

Recall that a topological group $G$ is called sequentially complete if every Cauchy sequence has a limit point. A locally convex space $E$ is called weakly sequentially complete if the space $E_{w}$ is sequentially complete. The following assertion is well known for Banach spaces, we give its proof only for the sake of completeness and the reader convenience.

Proposition 2.4. Let $(E, \tau)$ be an lcs with the Schur property. Then $E$ is sequentially complete if and only if it is weakly sequentially complete.

Proof. Assume that $E$ is sequentially complete. Let $\left\{g_{n}\right\}_{n \in \mathbf{N}}$ be a Cauchy sequence in $E_{w}$. Then for any two strictly increasing sequences $\left\{m_{i}\right\}_{i \in \mathbf{N}}$ and $\left\{k_{i}\right\}_{i \in \mathbf{N}}$ in $\mathbf{N}$, the sequence $\left\{g_{m_{i}}-g_{k_{i}}\right\}_{i \in \mathbf{N}}$ weakly converges to $0 \in E$. By the Schur property $\left\{g_{m_{i}}-g_{k_{i}}\right\}_{i \in \mathbf{N}}$ converges to zero in $\tau$. So $\left\{g_{n}\right\}_{n \in \mathbf{N}}$ is a Cauchy sequence in $E$ and hence it converges to some $g \in E$. Therefore $g_{n} \rightarrow g$ in $\sigma\left(E, E^{\prime}\right)$. Thus $E$ is weakly sequentially complete.

Conversely, let $E$ be weakly sequentially complete. If $\left\{g_{n}\right\}_{n \in \mathbf{N}}$ is a Cauchy sequence in $E$, then it is a Cauchy sequence in $\sigma\left(E, E^{\prime}\right)$ and hence weakly converges to some $g \in E$. By the Schur property, $g_{n} \rightarrow g$ in the original topology $\tau$. Thus $E$ is sequentially complete.

For a nonzero $x=\left(x_{n}\right) \in \ell_{\infty}$, we denote by $\operatorname{supp}(x):=\left\{n \in \mathbf{N}: x_{n} \neq 0\right\}$ the support of $x$. For every $n \in \mathbf{N}$, set $e_{n}:=(0, \ldots, 0,1,0, \ldots)$, where 1 is placed in position $n$. We shall consider the sequence $\left\{e_{n}: n \in \mathbf{N}\right\}$ also as the (standard) unit basis of $\ell_{1}$. The span of a subset $A$ of an lcs $E$ is denoted by $\operatorname{span}(A)$.

We shall say that a sequence $A=\left\{a_{n}\right\}_{n \in \mathbf{N}}$ of an lcs $E$ is equivalent to the unit basis $\left\{e_{n}: n \in \mathbf{N}\right\}$ of $\ell_{1}$ if there exists a linear topological isomorphism $R$ from the closure of $\operatorname{span}(A)$ onto a subspace of $\ell_{1}$ such that $R\left(a_{n}\right)=e_{n}$ for every $n \in \mathbf{N}$ (we do not assume that the closure of $\operatorname{span}(A)$ is complete).

Lemma 2.5. Let $A=\left\{a_{n}\right\}_{n \in \mathbf{N}}$ be a sequence in an lcs $E$ which is equivalent to the unit basis of $\ell_{1}$. Then $A$ is not weakly functionally bounded. 
Proof. It is well known that there exists an isometric isomorphism $T$ from $\ell_{1}$ into $\ell_{\infty}$. We claim that the sequence $S:=\left\{T\left(e_{n}\right): n \in \mathbf{N}\right\}$ is not weakly functionally bounded in $\ell_{\infty}$. Indeed, suppose for a contradiction that $S$ is weakly functionally bounded in $\ell_{\infty}$. Since $\left(\ell_{\infty}\right)_{w}$ is a $\mu$-space by [40], the weak closure $K$ of $S$ is a weakly compact subset of $\ell_{\infty}$. As $\left(\ell_{1}\right)_{w}$ is a closed subspace of $\left(\ell_{\infty}\right)_{w}$, we obtain that $K$ is compact in $\left(\ell_{1}\right)_{w}$. Therefore $K$ is norm compact by the Schur property of $\ell_{1}$, a contradiction. Thus $S$ is not weakly functionally bounded in $\ell_{\infty}$.

Now let $M$ be the closure of $\operatorname{span}(A)$ in $E$ and let $R$ be a linear topological isomorphism of $M$ onto a subspace of $\ell_{1}$ such that $R\left(a_{n}\right)=e_{n}$ for every $n \in \mathbf{N}$. The injectivity of $\ell_{\infty}$ (see Theorem 10.1.2 of [34]) implies that the operator $T \circ R$ can be extended to a continuous operator $Q$ from $E$ to $\ell_{\infty}$. Since $Q$ is also weakly continuous, the claim implies that $A$ is not weakly functionally bounded in $E$.

Following [22], a locally convex space $E$ is said to have the Rosenthal property if every bounded sequence in $E$ has a subsequence which either (1) is Cauchy in the weak topology, or (2) is equivalent to the unit basis of $\ell_{1}$.

Proposition 2.6. Let $(E, \tau)$ be a sequentially complete lcs with the Rosenthal property. If $E$ has the Schur property, then every functionally bounded subset $A$ of $E_{w}$ is relatively sequentially compact in $E$.

Proof. We have to show that every sequence $\left\{a_{n}\right\}_{n \in \mathbf{N}} \subseteq A$ has a convergent subsequence in $E$. Clearly, $A$ is a bounded subset of $E$. Hence, by the Rosenthal property, $\left\{a_{n}\right\}_{n \in \mathbf{N}}$ has a subsequence $\left\{b_{n}\right\}_{n \in \mathbf{N}}$ which either (1) is Cauchy in the weak topology $\tau_{w}$, or (2) is equivalent to the unit basis of $\ell_{1}$. The case (2) is not fulfilled by Lemma 2.5 , so $\left\{b_{n}\right\}_{n \in \mathbf{N}}$ is weakly Cauchy. Then, by Proposition $2.4, b_{n}$ weakly converges to a point $b \in E$, and hence, by the Schur property, $b_{n} \rightarrow b$ in the original topology $\tau$. Thus $A$ is relatively sequentially compact in $E$.

Taking into account that every Banach space is sequentially complete and being motivated by the Rosenthal property and the Eberlein-Šmulyan theorem, we denote by RES the class of all sequentially complete locally convex spaces $E$ with the Rosenthal property such that the closure $\bar{A}$ of every relatively sequentially compact subset $A$ of $E$ is compact.

Theorem 2.7. If $(E, \tau) \in \mathrm{RES}$, then the following assertions are equivalent:

(i) $E$ has the Schur property;

(ii) there is $\mathcal{P} \in \mathfrak{P}_{0}$ such that $E$ weakly respects $\mathcal{P}$;

(iii) $E$ weakly respects $\mathcal{F B}$ and is a countably $\mu$-space;

(iv) $E$ weakly respects all $\mathcal{P} \in \mathfrak{P}$ and is a countably $\mu$-space;

(v) $E$ is a countably $\mu$-space and every bounded non-functionally bounded sequence in $E$ has a subsequence which is equivalent to the unit basis of $\ell_{1}$.

In these cases every weakly functionally bounded subset of $E$ is relatively compact in $E$ and the space $E_{w}$ is a $\mu$-space.

Proof. (i) $\Rightarrow$ (iv) Let $A \in \mathcal{P}\left(E_{w}\right)$ for some $\mathcal{P} \in \mathfrak{P}$. Then $A$ is a functionally bounded subset of $E_{w}$. By Proposition 2.6, $A$ is relatively sequentially compact in $E$. As $E \in \operatorname{RES}$ we obtain that the $\tau$-closure $\bar{A}$ of $A$ is $\tau$-compact, i.e., $A$ is relatively compact in $E$. Therefore the space $E_{w}$ and hence also $E$ are $\mu$-spaces, and $A \in \mathcal{F} \mathcal{B}(E)$. Thus $E$ weakly respects functional boundedness. If $\mathcal{P} \in \mathfrak{P}_{0}$ and $\bar{A}$ is $\tau$-compact, the identity map $\left(\bar{A},\left.\tau\right|_{\bar{A}}\right) \rightarrow\left(\bar{A},\left.\tau_{w}\right|_{\bar{A}}\right)$ is a homeomorphism and hence $A \in \mathcal{P}(E)$. Thus $E$ weakly respects $\mathcal{P}$. 
(ii) $\Rightarrow$ (i) follows from Proposition 2.2. The implications (i) $\Rightarrow$ (ii), (iv) $\Rightarrow$ (i) and (iv) $\Rightarrow$ (iii) are clear.

(iii) $\Rightarrow$ (i) Let $S$ be a convergent sequence in $E_{w}$ with the limit point. Then $S \in \mathcal{F B}\left(E_{w}\right)$, and hence $S$ is functionally bounded in $E$. As $E$ is a countably $\mu$-space, the closed countable subset $S$ of $E$ is compact. Since the identity map $\left(S,\left.\tau\right|_{S}\right) \rightarrow\left(S,\left.\tau_{w}\right|_{S}\right)$ is a homeomorphism, we obtain that $S$ is a convergent sequence in $E$. Thus $E$ has the Schur property.

(i),(iii) $\Rightarrow$ (v) Let $S=\left\{g_{n}\right\}_{n \in \mathbf{N}}$ be a bounded non-functionally bounded sequence in $E$. Observe that $S$ is also not functionally bounded in $E_{w}$ since $E$ weakly respects functional boundedness. Take a continuous real-valued function $f$ on $E_{w}$ which is unbounded on $S$ and take a subsequence $S^{\prime}=\left\{g_{n_{k}}\right\}_{k \in \mathbf{N}}$ of $S$ such that $f\left(g_{n_{k}}\right) \rightarrow \infty$. We claim that $S^{\prime}$ does not have a weakly Cauchy subsequence $S^{\prime \prime}$. Indeed, otherwise, the weak sequential completeness of $E$ (see Proposition 2.4) would imply that $S^{\prime \prime}$ converges in $\tau_{w}$. Hence $f$ is bounded on $S^{\prime \prime}$ that is impossible since $f\left(g_{n_{k}}\right) \rightarrow \infty$. Finally, the Rosenthal property implies that $S$ has a subsequence which is equivalent to the unit basis of $\ell_{1}$.

$(\mathrm{v}) \Rightarrow(\mathrm{i})$ Let $S$ be a weakly convergent sequence in $E$ with the limit point. Then $S$ is bounded. We claim that $S$ is functionally bounded in $E$. Indeed, otherwise, $S$ would contain a subsequence $S^{\prime}$ which is equivalent to the unit basis of $\ell_{1}$. Therefore $S$ is not weakly functionally bounded by Lemma 2.5 , a contradiction. Now since $E$ is a countably $\mu$-space, the closed countable subset $S$ of $E$ is compact. As the identity $\operatorname{map}\left(S,\left.\tau\right|_{S}\right) \rightarrow\left(S,\left.\tau_{w}\right|_{S}\right)$ is a homeomorphism, we obtain that $S$ is a convergent sequence in $E$. Thus $E$ has the Schur property.

Being motivated by a result of Hernández and Macario [27, Theorem 3.2], we prove the following sufficiently general result. Recall that a subset $A$ of a topological space $(X, \tau)$ is called $C$-embedded $\left(C^{*}\right.$-embedded) if every continuous (respectively, bounded) real-valued function $f$ on $\left(A,\left.\tau\right|_{A}\right)$ can be extended to a continuous function $\bar{f}$ on $X$.

Theorem 2.8. If an lcs $(E, \tau)$ is such that $E_{w}$ is a $\mu$-space, then the following assertions are equivalent:

(i) E weakly respects compactness;

(ii) $E$ weakly respects countable compactness and $E$ is a $\mu$-space;

(iii) $E$ weakly respects pseudocompactness and $E$ is a $\mu$-space;

(iv) $E$ weakly respects functional boundedness and $E$ is a $\mu$-space;

(v) $E$ is a $\mu$-space and every non-functionally bounded subset $A$ of $E$ has an infinite subset $B$ which is discrete and $C$-embedded in $E_{w}$.

If (i)-(v) hold, then every functionally bounded subset in $E_{w}$ is relatively compact in $E$.

Proof. (i) $\Rightarrow$ (ii) Let $A$ be a countably compact subset of $E_{w}$. As $E_{w}$ is a $\mu$-space, the $\tau_{w}$-closure $\bar{A}$ of $A$ is compact in $E_{w}$. Therefore $\bar{A}$ is compact in $E$, and hence $A$ is relatively compact in $E$. Since the identity map $\left(\bar{A},\left.\tau\right|_{\bar{A}}\right) \rightarrow\left(\bar{A},\left.\tau_{w}\right|_{\bar{A}}\right)$ is a homeomorphism, we obtain that $A$ is countably compact in $E$. Thus $E$ respects countable compactness. The same proof shows that every functionally bounded subset in $E_{w}$ is relatively compact in $E$, and in particular $E$ is a $\mu$-space.

(ii) $\Rightarrow$ (iii) Let $A$ be a pseudocompact subset of $E_{w}$. Then the closure $K$ of $A$ in $E_{w}$ is $\tau_{w}$-compact because $E_{w}$ is a $\mu$-space. Therefore $K$ is countably compact in $E$. Being closed $K$ also is compact in $E$ since $E$ is a $\mu$-space. As the identity map 
$\left(K,\left.\tau\right|_{K}\right) \rightarrow\left(K,\left.\tau_{w}\right|_{K}\right)$ is a homeomorphism, we obtain that $A$ is pseudocompact in $E$. Thus $E$ weakly respects pseudocompactness.

The implication (iii) $\Rightarrow$ (iv) is proved analogously to (ii) $\Rightarrow$ (iii).

(iv) $\Rightarrow$ (v) Let $A$ be a non-functionally bounded subset of $E$. As $E$ weakly respects functional boundedness it follows that $A$ is not functionally bounded in $E_{w}$. Let $f$ be a continuous real-valued function on $E_{w}$ which is unbounded on $A$. If we take $B$ as a sequence $\left\{a_{n}\right\}_{n \in \mathbf{N}}$ in $A$ such that $\left|f\left(a_{n+1}\right)\right|>\left|f\left(a_{n}\right)\right|+1$ for all $n \in \mathbf{N}$, then $B$ is discrete and $C$-embedded in $E_{w}$.

$(\mathrm{v}) \Rightarrow$ (i) Let $K$ be a compact subset of $E_{w}$. Then $K$ must be functionally bounded in $E$. Since $E$ is a $\mu$-space and $K$ is also closed in $E$ we obtain that $K$ is compact in $E$. Thus $E$ weakly respects compactness.

We need also the following mild completeness type property. An lcs $E$ is said to have the cp-property if every countable precompact subset of $E$ has compact closure. Clearly, any complete lcs has the cp-property, and each lcs with the cp-property is a countably $\mu$-space.

Lemma 2.9. Let $E$ be a locally convex space. Then:

(i) every functionally bounded subset $A$ of $E$ is precompact;

(ii) if $E$ has the cp-property, then a separable subset $B$ of $E$ is functionally bounded if and only if $B$ is precompact.

Proof. (i) If $A$ is not precompact, Theorem 5 of [3] implies that $A$ has an infinite uniformly discrete subset $C$, i.e., there is a neighborhood $U$ of zero in $E$ such that $c-c^{\prime} \notin U$ for every distinct $c, c^{\prime} \in C$. Thus, by Lemma 2.1 of [19], $C$ and hence also $A$ are not functionally bounded, a contradiction.

(ii) follows from (i) and the cp-property.

In [11, Lemma 3] Díaz extends the Rosenthal $\ell_{1}$ theorem to all Fréchet spaces. A much more general result was obtained recently by Ruess in [39]. Recall that an lcs $E$ is locally complete if every closed disc in $E$ is a Banach disc; every sequentially complete lcs is locally complete by Corollary 5.1 .8 of [35].

Theorem 2.10. (Ruess) Every locally complete lcs $E$ whose every separable bounded set is metrizable has the Rosenthal property.

Below we give some examples of locally convex spaces which belong to the class RES and have the cp-property. An lcs $(E, \tau)$ is called an $(L M)$-space and write $E=$ $\underset{\lim _{n}}{\longrightarrow} E_{n}$ if there is a sequence $\left\{\left(E_{n}, \tau_{n}\right)\right\}_{n \in \mathbf{N}}$ of metrizable locally convex spaces such that $\left(E_{n}, \tau_{n}\right)$ is continuously included in $\left(E_{n+1}, \tau_{n+1}\right)$, and $\tau$ is the finest Hausdorff locally convex topology on $E=\bigcup_{n} E_{n}$ such that $\left(E_{n}, \tau_{n}\right)$ is continuously included in $(E, \tau)$. If in addition all the spaces $\left(E_{n}, \tau_{n}\right)$ are Fréchet spaces and $\left.\tau_{n+1}\right|_{E_{n}}=\tau_{n}$ holds for every $n \in \mathbf{N}$, the space $E$ is called a strict $(L F)$-space.

Proposition 2.11. A locally convex space $(E, \tau)$ belongs to RES and has the cp-property if one of the following conditions holds:

(i) $E$ is quasi-complete and every separable bounded subset of $E$ is metrizable;

(ii) $E$ is a strict $(L F)$-space (in particular, a Fréchet space);

(iii) $E$ is the strong dual $H_{\beta}^{\prime}$ of a quasinormable metrizable lcs $H$.

Proof. (i) Let $A$ be a precompact subset of $E$. Then $A$ is bounded, and since $E$ is quasi-complete, the closure $\bar{A}$ of $A$ is complete. Therefore $\bar{A}$ is compact by Theorem 3.4.1 of [34]. Thus $E$ has the cp-property. The space $E$ has the Rosenthal property by Theorem 2.10. So to show that $E$ belongs to the class RES we have 
to prove that the closure $\bar{B}$ of a relatively sequentially compact subset $B$ of $E$ is compact. It is easy to see that $B$ is functionally bounded in $E$. Therefore $B$ is precompact by (i) of Lemma 2.9. As we proved above, $\bar{B}$ is compact.

(ii) Let $E=\underline{\lim } E_{n}$, where all the $E_{n}$ are Fréchet spaces. Note that, for every $n \in \mathbf{N}$, the space $\overrightarrow{E_{n}}$ is a closed subspace of $E$. Theorem 12.1 .10 of [34] implies that $E$ is complete. By Theorem 12.1.7 of [34], any bounded subset of $E$ sits in some $E_{n}$ and hence is metrizable. Now (i) applies.

(iii) The space $E$ is quasi-complete by Proposition 11.2.3 of [30], and Theorem 2 (see also the diagram before this theorem) of [4] implies that every bounded subset of $E$ is metrizable. Now (i) applies.

Now we are ready to prove the main result of the paper.

Proof of Theorem 1.2. As we showed in the proof of (i) in Proposition 2.11, the quasi-complete space $E$ is a $\mu$-space and has the cp-property. Also the space $E_{w}$ is a $\mu$-space by [40]. Now the theorem follows from Theorems 2.7 and 2.8 and Lemma 2.9 .

Let $X$ and $Y$ be Tychonoff spaces. The space $C(X, Y)$ of all continuous functions from $X$ to $Y$ endowed with the compact-open topology $\tau_{k}$ is denoted by $C_{k}(X, Y)$. If $Y=\mathbf{R}$, we set $C(X):=C(X, \mathbf{R})$ and $C_{k}(X):=C_{k}(X, \mathbf{R})$. The sets

$$
[K ; \varepsilon]:=\{f \in C(X):|f(x)|<\varepsilon \forall x \in K\}
$$

where $K$ is a compact subset of $X$ and $\varepsilon>0$, form a base at zero of $\tau_{k}$.

Let $E$ be an lcs over the field $\mathbf{F}$ of real numbers $\mathbf{R}$ or complex numbers $\mathbf{C}$. We denote by $\mu\left(E, E^{\prime}\right)$ the Mackey topology on $E$ and set $E_{\beta}^{\prime}:=\left(E^{\prime}, \beta\left(E^{\prime}, E\right)\right)$, where $\beta\left(E^{\prime}, E\right)$ is the strong topology on $E^{\prime}$. If $A$ is a subset of $E$, we denote by $A^{\circ}$ and $\overline{\operatorname{acx}}(A)$ the polar of $A$ and the closed absolutely convex hull of $A$, respectively. The Krein theorem $[32, \S 24.5(4)]$ states that if $K$ is a weakly compact subset of $E$, then $\overline{\operatorname{acx}}(K)$ is weakly compact if and only if $\overline{\operatorname{acx}}(K)$ is $\mu\left(E, E^{\prime}\right)$-complete. We shall say that $E$ has the Krein property or is a Krein space if $\overline{\operatorname{acx}}(K)$ is weakly compact for every weakly compact subset $K$ of $E$. Therefore $E$ is a Krein space if $\left(E, \mu\left(E, E^{\prime}\right)\right)$ is quasi-complete. In particular, every quasibarrelled quasi-complete space is a Krein space. By the definition of $\mu\left(E^{\prime}, E\right)$, the Mackey topology $\mu\left(E^{\prime}, E\right)$ on $E^{\prime}$ is always weaker than the restriction $\left.\tau_{k}\right|_{E^{\prime}}$ of the compact-open topology $\tau_{k}$ of $C_{k}\left(E_{w}, \mathbf{F}\right)$ onto $E^{\prime}$. Below we show that the equality $\mu\left(E^{\prime}, E\right)=\left.\tau_{k}\right|_{E^{\prime}}$ characterizes Krein spaces.

Proposition 2.12. Let $E$ be an lcs over $\mathbf{F}=\mathbf{R}$ or $\mathbf{C}$. Then:

(i) $E$ is a Krein space if and only if $\left(E^{\prime}, \mu\left(E^{\prime}, E\right)\right)$ is a subspace of $C_{k}\left(E_{w}, \mathbf{F}\right)$;

(ii) if $E$ is quasi-complete and weakly respects compactness, then $\left(E^{\prime}, \mu\left(E^{\prime}, E\right)\right)$ is a subspace of $C_{k}(E, \mathbf{F})$;

(iii) if $E$ is a quasi-complete Mackey space, then $E$ weakly respects compactness if and only if $\left(E^{\prime}, \mu\left(E^{\prime}, E\right)\right)$ is a subspace of $C_{k}(E, \mathbf{F})$.

Proof. (i) Assume that $E$ is a Krein space. Fix a weakly compact subset $K$ of $E$. Then $\overline{\operatorname{acx}}(K)$ is also weakly compact by the Krein property. Therefore $\mu\left(E^{\prime}, E\right) \geq$ $\left.\tau_{k}\right|_{E^{\prime}}$. Thus $\mu\left(E^{\prime}, E\right)=\left.\tau_{k}\right|_{E^{\prime}}$ and hence $\left(E^{\prime}, \mu\left(E^{\prime}, E\right)\right)$ is a subspace of $C_{k}\left(E_{w}, \mathbf{F}\right)$. Conversely, assume that $\left(E^{\prime}, \mu\left(E^{\prime}, E\right)\right)$ is a subspace of $C_{k}\left(E_{w}, \mathbf{F}\right)$. Fix a weakly compact subset $K$ of $E$. Then there exists an absolutely convex weakly compact subset $C$ of $E$ such that $C^{\circ} \subseteq K^{\circ}$. Therefore $K \subseteq K^{\circ \circ} \subseteq C^{\circ \circ}=C$. Thus $\overline{\operatorname{acx}}(K)$ is a weakly compact subset of $E$. 
(ii) If $K$ is an absolutely convex weakly compact subset of $E$, then $K$ is compact in $E$. Therefore $\mu\left(E^{\prime}, E\right) \leq\left.\tau_{k}\right|_{E^{\prime}}$. Conversely, for every compact subset $K$ of $E$, the quasi-completeness of $E$ implies that the closed absolutely convex hull of $K$ is also compact in $E$. Thus $\mu\left(E^{\prime}, E\right) \geq\left.\tau_{k}\right|_{E^{\prime}}$ and hence $\mu\left(E^{\prime}, E\right)=\left.\tau_{k}\right|_{E^{\prime}}$.

(iii) The necessity follows from (ii). To prove sufficiency, let $K$ be a weakly compact subset of $E$. As $E$ is a Krein space, the set $K_{0}:=\overline{\operatorname{acx}}(K)$ is also weakly compact. By assumption there is a compact subset $C_{0}$ of $E$ such that $C_{0}^{\circ} \subseteq K_{0}^{\circ}$. Since $E$ is quasi-complete, we obtain that the set $C:=\overline{\operatorname{acx}}\left(C_{0}\right)$ is also a compact subset of $E$. Then $K \subseteq K^{\circ \circ} \subseteq C_{0}^{\circ \circ} \subseteq C^{\circ \circ}=C$. Therefore $K$ being closed in $E$ is a compact subset of $E$. Thus $E$ weakly respects compactness.

The class of strict $(L F)$-spaces is one of the most important classes of locally convex spaces. Below we apply Theorem 1.2 and Proposition 2.12 to characterize strict $(L F)$-spaces with the Schur property.

Corollary 2.13. Let $E=\lim _{\longrightarrow} E_{n}$ be a strict $(L F)$-space over $\mathbf{F}=\mathbf{R}$ or $\mathbf{C}$. Then the following assertions are equivalent:

(i) there is a $\mathcal{P} \in \mathfrak{P}$ such that $E$ weakly respects $\mathcal{P}$;

(ii) $E$ weakly respects all $\mathcal{P} \in \mathfrak{P}$;

(iii) every bounded non-precompact sequence in $E$ has a subsequence which is equivalent to the unit basis of $\ell_{1}$;

(iv) there is a $\mathcal{P} \in \mathfrak{P}$ such that all the spaces $E_{n}$ weakly respect $\mathcal{P}$;

(v) for every $n \in \mathbf{N}$, the space $E_{n}$ weakly respects all $\mathcal{P} \in \mathfrak{P}$;

(vi) every non-precompact bounded subset of $E$ has an infinite subset which is discrete and $C$-embedded in $E_{w}$;

(vii) $\left(E^{\prime}, \mu\left(E^{\prime}, E\right)\right)$ is a subspace of $C_{k}(E, \mathbf{F})$.

Proof. The equivalences (i) $\Leftrightarrow$ (ii) $\Leftrightarrow$ (iii) $\Leftrightarrow$ (vi) follow from Theorem 1.2 and (ii) of Proposition 2.11.

(iii) $\Rightarrow(\mathrm{v})$ Fix $n \in \mathbf{N}$ and let $S$ be a bounded non-precompact sequence in $E_{n}$. As $E_{n}$ is a closed subspace of $E$, there is a subsequence $S^{\prime}$ of $S$ which is equivalent to the unit basis of $\ell_{1}$. Clearly, the closure of $\operatorname{span}\left(S^{\prime}\right)$ in $E$ is contained in $E_{n}$. Therefore $E_{n}$ respects all the properties $\mathcal{P} \in \mathfrak{P}$ by Theorem 1.2 and (ii) of Proposition 2.11.

(v) $\Rightarrow$ (iv) is trivial. To prove the implication (iv) $\Rightarrow$ (iii), let $S$ be a bounded nonprecompact sequence in $E$. Then $S \subseteq E_{n}$ for some $n \in \mathbf{N}$. Applying Theorem 1.2 and (ii) of Proposition 2.11 to the Fréchet space $E_{n}$, we obtain that $S$ has a subsequence $S^{\prime}$ which is equivalent to the unit basis of $\ell_{1}$. It remains to note that $E_{n}$ is a closed subspace of $E$.

Taking into account that $E$ is a complete Mackey space (see [34, Theorem 12.1.10] and [30, Corollary 8.8.11]), (ii) and (vii) are equivalent by (iii) of Proposition 2.12 and Theorem 1.2.

Let $K$ be an infinite compact space. Then the Banach space $C(K)$ does not have the Schur property since it contains an isomorphic copy of $c_{0}$, see Theorem 14.26 of [18]. The next proposition (which, perhaps, is known but hard to find explicitly stated) generalizes this result.

Proposition 2.14. For a Tychonoff space $X$, the space $C_{k}(X)$ has the Schur property if and only if $X$ does not contain an infinite compact subset.

Proof. Let $C_{k}(X)$ have the Schur property. Suppose for a contradiction that $X$ contains an infinite compact subset $K$. By Lemma 11.7.1 of [30], take a oneto-one sequence $\left\{x_{n}\right\}_{n \in \mathbf{N}}$ in $K$ and a sequence $\left\{U_{n}\right\}_{n \in \mathbf{N}}$ of open subsets of $X$ such 
that $x_{n} \in U_{n}$ for each $n \in \mathbf{N}$ and $\overline{U_{n}} \cap \overline{U_{m}}=\emptyset$ for every distinct $n, m \in \mathbf{N}$. For every $n \in \mathbf{N}$, take a continuous function $f_{n}: X \rightarrow[0,1]$ with support in $U_{n}$ and such that $f_{n}\left(x_{n}\right)=1$. We claim that $f_{n}$ weakly converges to zero. Indeed, fix a regular Borel measure $\mu \in M_{c}(X)=C_{k}(X)^{\prime}$ with compact support. We can assume that $\mu$ is positive. Since $\mu$ is finite and regular, observe that $\mu\left(U_{n}\right) \rightarrow 0$. Then $0 \leq \mu\left(f_{n}\right) \leq \mu\left(U_{n}\right) \rightarrow 0$. This proves the claim. On the other hand, by construction we have $f_{n} \notin[K ; 1 / 3]$ for every $n \in \mathbf{N}$. Therefore $f_{n} \not \rightarrow 0$ in the compact-open topology. Thus $C_{k}(X)$ does not have the Schur property.

Conversely, if $X$ does not contain infinite compact subsets, then the compactopen topology $\tau_{k}$ coincides with the pointwise topology and hence $\left(C_{k}(X)\right)_{w}=$ $C_{k}(X)$. Thus $C_{k}(X)$ trivially has the Schur property.

\section{The Schur property and Dunford-Pettis type properties}

In this section we give concrete constructions of Schur spaces and extend some known results for Banach and Fréchet spaces to larger classes of locally convex spaces.

Recall that a Tychonoff space $X$ is called an angelic space if (1) every relatively countably compact subset of $X$ is relatively compact, and (2) any compact subspace of $X$ is Fréchet-Urysohn. Note that any subspace of an angelic space is angelic, and a subset $A$ of an angelic space $X$ is compact if and only if it is countably compact if and only if $A$ is sequentially compact, see Lemma 0.3 of [37]. Being motivated by the last property we say that a Tychonoff space $X$ is sequentially angelic if a subset $K$ of $X$ is compact if and only if $K$ is sequentially compact.

Recall that an lcs $E$ has the Dunford-Pettis property ((DP) property for short) if every absolutely convex $\sigma\left(E, E^{\prime}\right)$-compact subset of $E$ is precompact for the topology $\tau_{\Sigma^{\prime}}$ of uniform convergence on the absolutely convex, equicontinuous, $\sigma\left(E^{\prime}, E^{\prime \prime}\right)$ compact subset of $E^{\prime}$ (see $\S 9.4$ of [16]); $E$ has the Grothendieck property if every weak-* convergent sequence in $E^{\prime}$ is weakly convergent. Recall also (see [7]) that a Banach space $E$ has the $*$-Dunford-Pettis property $(*-(D P)$ property) if given a weakly null sequence $\left\{x_{n}\right\}_{n \in \mathbf{N}}$ in $E$ and a weakly-* null sequence $\left\{\chi_{n}\right\}_{n \in \mathbf{N}}$ in $E^{\prime}$, then $\lim _{n} \chi_{n}\left(x_{n}\right)=0$. Analogously we say that an lcs $E$ has

- the sequential Dunford-Pettis property $((s D P)$ property) if given weakly null sequences $\left\{x_{n}\right\}_{n \in \mathbf{N}}$ and $\left\{\chi_{n}\right\}_{n \in \mathbf{N}}$ in $E$ and $E_{\beta}^{\prime}$, respectively, then $\lim _{n} \chi_{n}\left(x_{n}\right)=$ 0 ;

- the $*$-sequential Dunford-Pettis property $(*-(s D P)$ property) if given a weakly null sequence $\left\{x_{n}\right\}_{n \in \mathbf{N}}$ in $E$ and a weakly-* null sequence $\left\{\chi_{n}\right\}_{n \in \mathbf{N}}$ in $E^{\prime}$, then $\lim _{n} \chi_{n}\left(x_{n}\right)=0$.

Clearly, the $*-(s D P)$ property implies the $(s D P)$ property, but the converse is not true in general as the Banach space $c_{0}$ shows. Any Banach space $E$ with the $*-(D P)$ property contains an isomorphic copy of $\ell_{1}$, see [29, Proposition 4]. It is easy to see that every Banach space $E$ with the Schur property has the $*-(s D P)$ property, but in general the converse is false (for example $E=\ell_{\infty}$ ). Proposition 3.3 of [1] implies that: $(1)$ every barrelled space with the $(D P)$-property has the $(s D P)$ property, and $(2)$ if both $E$ and $E_{\beta}^{\prime}$ are sequentially angelic and $E$ has the $(s D P)$ property, then $E$ has the $(D P)$ property. Moreover, if $E$ is a strict $(L F)$-space, then $E$ has the $(D P)$ property if and only if it has the $(s D P)$-property, see Corollary 3.4 of [1]. Banach spaces with the $*-(D P)$ property are studied in [9]. 
The next proposition generalizes the corresponding well known results for Banach spaces. Recall that an lcs $E$ is called $c_{0}$-barrelled if every $\sigma\left(E^{\prime}, E\right)$-null sequence in $E^{\prime}$ is equicontinuous.

Proposition 3.1. Let $E$ be a locally convex space. Then:

(i) if $E$ is $c_{0}$-barrelled and has the Schur property, then $E$ has the *-(sDP) property;

(ii) if $E_{\beta}^{\prime}$ has the Schur property, then $E$ has the (sDP) property;

(iii) if $E$ is quasibarrelled and $E_{\beta}^{\prime}$ has the $(s D P)$ property, then also $E$ has the $(s D P)$ property.

Proof. (i) Let $\left\{x_{n}\right\}_{n \in \mathbf{N}}$ be a weakly null sequence in $E$ and $\left\{\chi_{n}\right\}_{n \in \mathbf{N}}$ be a weakly* null sequence in $E_{\beta}^{\prime}$. As $E$ is $c_{0}$-barrelled, there is a neighborhood $U$ of zero in $E$ such that $\left\{\chi_{n}\right\}_{n \in \mathbf{N}} \subseteq U^{\circ}$. By the Schur property, for each $\varepsilon>0$ choose $N \in \mathbf{N}$ such that $x_{n} \in \varepsilon U$ for every $n>N$. Then $\left|\chi_{n}\left(x_{n}\right)\right| \leq \varepsilon$ for $n>N$. Thus $\chi_{n}\left(x_{n}\right) \rightarrow 0$ and $E$ has the $*-(s D P)$ property.

(ii) Let $\left\{x_{n}\right\}_{n \in \mathbf{N}}$ and $\left\{\chi_{n}\right\}_{n \in \mathbf{N}}$ be weakly null sequences in $E$ and $E_{\beta}^{\prime}$, respectively. Since $E_{\beta}^{\prime}$ has the Schur property, $\chi_{n} \rightarrow 0$ in the strong topology $\beta\left(E^{\prime}, E\right)$ on $E^{\prime}$. Clearly, $S=\left\{x_{n}\right\}_{n \in \mathbf{N}}$ is a bounded subset of $E$. Therefore for each $\varepsilon>0$ there is an $N \in \mathbf{N}$ such that $\left|\chi_{n}(x)\right|<\varepsilon$ for every $x \in S$ and every $n>N$. Thus $\lim _{n} \chi_{n}\left(x_{n}\right)=0$.

(iii) Let $\left\{x_{n}\right\}_{n \in \mathbf{N}}$ and $\left\{\chi_{n}\right\}_{n \in \mathbf{N}}$ be weakly null sequences in $E$ and $E_{\beta}^{\prime}$, respectively. Since $E$ is quasibarrelled, it is a subspace of $\left(E_{\beta}^{\prime}\right)_{\beta}^{\prime}$ by Theorem 15.2.3 of [34]. So $x_{n} \rightarrow 0$ also in $\sigma\left(E^{\prime \prime}, E^{\prime \prime \prime}\right)$. Now the $(s D P)$ property of $E_{\beta}^{\prime}$ implies $\chi_{n}\left(x_{n}\right) \rightarrow 0$. Thus $E$ has the $(s D P)$ property.

Remark 3.2. Pethe and Thakare showed in [36] that the Banach dual $E^{\prime}$ of a Banach space $E$ has the Schur property if and only if $E$ has the Dunford-Pettis property and does not contain an isomorphic copy of $\ell_{1}$.

Proposition 3.3. Let $E$ be an lcs with the $(s D P)$ property such that $E_{w}$ is sequentially angelic.

(i) Every $\sigma\left(E^{\prime}, E^{\prime \prime}\right)$-null sequence $\left\{\chi_{n}\right\}_{n \in \mathbf{N}}$ in $E^{\prime}$ converges to zero also in $\mu\left(E^{\prime}, E\right)$. Consequently, every $\sigma\left(E^{\prime}, E^{\prime \prime}\right)$-sequentially compact subset of $E^{\prime}$ is also $\mu\left(E^{\prime}, E\right)$ sequentially compact.

(ii) If additionally $E$ has the Grothendieck property, then $\left(E^{\prime}, \mu\left(E^{\prime}, E\right)\right)$ and $\left(E^{\prime}, \sigma\left(E^{\prime}, E^{\prime \prime}\right)\right)$ have the same sequentially compact sets. Moreover, the space $H:=\left(E^{\prime}, \mu\left(E^{\prime}, E\right)\right)$ has the Schur property.

Proof. (i) (cf. [14, 19.18(c)]) Suppose for a contradiction that $\chi_{n} \not \rightarrow 0$ in $\mu\left(E^{\prime}, E\right)$. Then there is a standard $\mu\left(E^{\prime}, E\right)$-neighborhood $[K ; \varepsilon]=\left\{\chi \in E^{\prime}:|\chi(x)|<\varepsilon \forall x \in\right.$ $K$ \} of zero in $E^{\prime}$, where $K$ is an absolutely convex compact subset of $E_{w}$, which does not contain a subsequence $\left\{\chi_{n_{k}}\right\}_{k \in \mathbf{N}}$ of $\left\{\chi_{n}\right\}_{n \in \mathbf{N}}$. Therefore, for every $k \in \mathbf{N}$ one can find $x_{k} \in K$ such that $\left|\chi_{n_{k}}\left(x_{k}\right)\right| \geq \varepsilon$. Since $E_{w}$ is sequentially angelic, $K$ is sequentially compact and hence we can assume that $x_{k}$ weakly converges to $x \in K$. Then the $(s D P)$ property implies

$$
\varepsilon \leq\left|\chi_{n_{k}}\left(x_{k}\right)\right| \leq\left|\chi_{n_{k}}(x)\right|+\left|\chi_{n_{k}}\left(x_{k}-x\right)\right| \rightarrow 0,
$$

a contradiction.

(ii) By (i) we have to show only that every sequentially compact subset $K$ of $\left(E^{\prime}, \mu\left(E^{\prime}, E\right)\right)$ is also $\sigma\left(E^{\prime}, E^{\prime \prime}\right)$-sequentially compact. Let $S=\left\{\chi_{n}: n \in \mathbf{N}\right\}$ be a 
sequence in $K$. Then there is a subsequence $S^{\prime}=\left\{\chi_{n_{k}}: k \in \mathbf{N}\right\}$ of $S$ which $\mu\left(E^{\prime}, E\right)$ converges to some $\chi \in K$. Then $\chi_{n_{k}} \rightarrow \chi$ in $\sigma\left(E^{\prime}, E\right)$ and hence also in $\sigma\left(E^{\prime}, E^{\prime \prime}\right)$ by the Grothendieck property. Thus $K$ is $\sigma\left(E^{\prime}, E^{\prime \prime}\right)$-sequentially compact.

To prove that $H$ has the Schur property let $S$ be a $\sigma\left(E^{\prime}, E\right)$-null sequence. Then $S$ is a $\sigma\left(E^{\prime}, E^{\prime \prime}\right)$-null sequence by the Grothendieck property. Therefore, by (i), $S$ converges to zero in $\mu\left(E^{\prime}, E\right)$. Thus $H$ has the Schur property.

Let $E$ be a locally convex space. Recall that a subset $A$ of $E^{\prime}$ is called E-limited if

$$
\sup \left\{\left|\chi\left(x_{n}\right)\right|: \chi \in A\right\} \rightarrow 0
$$

whenever $\left\{x_{n}\right\}_{n \in \mathbf{N}}$ is a weakly null sequence in $E$. It is well known (see [25, Exercise 3.12]) that a Banach space $E$ has the Schur property if and only if the closed unit ball of the dual space $E^{\prime}$ is an $E$-limited set. Below we generalize this result to barrelled spaces.

Proposition 3.4. A barrelled space $(E, \tau)$ has the Schur property if and only if every $\sigma\left(E^{\prime}, E\right)$-bounded subset of $E^{\prime}$ is an E-limited set.

Proof. Assume that $E$ has the Schur property. Let $A$ be a $\sigma\left(E^{\prime}, E\right)$-bounded subset of $E^{\prime}$ and let $\left\{x_{n}\right\}_{n \in \mathbf{N}}$ be a $\sigma\left(E, E^{\prime}\right)$-null sequence in $E$. Since $E$ is barrelled there is a $\tau$-neighborhood $U$ of zero in $E$ such that $A \subseteq U^{\circ}$. For every $\varepsilon>0$, by the Schur property, there is an $N \in \mathbf{N}$ such that $x_{n} \in \varepsilon U$ for every $n>N$. Then $\left|\chi\left(x_{n}\right)\right|=\varepsilon\left|\chi\left((1 / \varepsilon) x_{n}\right)\right| \leq \varepsilon$ for every $\chi \in A$ and $n>N$. Thus $A$ is $E$-limited.

Conversely, assume that every $\sigma\left(E^{\prime}, E\right)$-bounded subset of $E^{\prime}$ is an $E$-limited set. Let $x_{n} \rightarrow 0$ in $\sigma\left(E, E^{\prime}\right)$ and let $U$ be an absolutely convex closed $\tau$-neighborhood of zero in $E$. Then there is an $N \in \mathbf{N}$ such that $\left|\chi\left(x_{n}\right)\right| \leq 1$ for every $\chi \in U^{\circ}$ and $n>$ $N$. So $x_{n} \in U^{\circ \circ}=U$ for every $n>N$. Thus $x_{n} \rightarrow 0$ in $\tau$ and $E$ has the Schur property.

Recall that a Tychonoff space $X$ is called an $F$-space if every cozero-set $A$ in $X$ is $C^{*}$-embedded. For numerous equivalent conditions for a Tychonoff space $X$ being an $F$-space see $[24,14.25]$. In particular, the Stone-Cech compactification $\beta \Gamma$ of a discrete space $\Gamma$ is a compact $F$-space.

In general the Schur property does not imply weak respecting compactness. Using the non-reflexivity of Banach spaces $C(K)$, it is shown in [42, Example 6 (p. 267)] or [14, Example 19.19] that the space $H=\left(E^{\prime}, \mu\left(E^{\prime}, E\right)\right)$, where $E=\ell_{\infty}(\mathbf{N})=C(\beta \mathbf{N})$, has the Schur property but does not weakly respect compactness. Below we generalize this result with a different proof.

Proposition 3.5. Let $K$ be an infinite compact $F$-space, $E=C(K)$ and set $H:=\left(E^{\prime}, \mu\left(E^{\prime}, E\right)\right)$. Then:

(i) $H$ is a complete space and has the Schur property;

(ii) $H$ does not weakly respect compactness.

Proof. (i) The space $H$ is complete by Exercise 3.41 of [18]. The Banach space $E$ has the $(s D P)$ property by Theorem 13.43 of [18] and has the Grothendieck property by Corollary 4.5.9 of [10]. Proposition 3.108 of [18] implies that $E_{w}$ is angelic. Thus $H$ has the Schur property by (ii) of Proposition 3.3.

(ii) Consider the closed unit ball $B^{*}$ in $E^{\prime}$. The Alaoglu theorem implies that $B^{*}$ is a weakly compact subset of $H$. Now assuming that $B^{*}$ is a compact subset 
of $H$ we apply Grothendieck's theorem [25, Theorem 3.11] to get that $B^{*}$ is $E$ limited. Therefore $E$ has the Schur property by Proposition 3.4. But this contradicts Proposition 2.14. Thus $H$ does not weakly respect compactness.

Valdivia [41] and Domanski and Drewnowski [13] proved independently that a Fréchet space $E$ does not contain $\ell_{1}$ if and only if every $\mu\left(E^{\prime}, E\right)$-null sequence in $E^{\prime}$ is strongly convergent to zero. In [39, Theorem 2.1] Ruess generalized this result.

Proposition 3.6 (Ruess). Let $E$ be a locally complete lcs whose every separable bounded set is metrizable. Then $E$ does not contain an isomorphic copy of $\ell_{1}$ if and only if every $\mu\left(E^{\prime}, E\right)$-null sequence in $E^{\prime}$ is strongly convergent to zero.

It is well known (see [30, Proposition 11.6.2]) that an lcs $E$ is Montel if and only if it is a quasi-complete quasibarrelled (equivalently, barrelled see [35, Corollary 5.1.10]) space and every equicontinuous set in $E^{\prime}$ is relatively compact for $\beta\left(E^{\prime}, E\right)$. It is proved in [6] (see also [5, Theorem 9]) that a Fréchet space $E$ is Montel if and only if every $\sigma\left(E^{\prime}, E\right)$-convergent sequence in $E^{\prime}$ is $\beta\left(E^{\prime}, E\right)$-convergent. Using Proposition 3.6 we obtain a similar result for a wider class of locally convex spaces.

Proposition 3.7. Let $E$ be an lcs whose every separable bounded set is metrizable. Then $E$ is a Montel space if and only if the following three conditions hold:

(i) $E$ is a quasi-complete quasibarrelled space;

(ii) every $\sigma\left(E^{\prime}, E\right)$-convergent sequence in $E^{\prime}$ is $\beta\left(E^{\prime}, E\right)$-convergent;

(iii) E has the Schur property.

Proof. Assume that $E$ is a Montel space. Then $E$ is a quasi-complete barrelled space by Proposition 11.6.2 of [30], and $E$ and $E_{\beta}^{\prime}$ have the Schur property by Proposition 2.3. The reflexivity of $E$ and the Schur property of $E_{\beta}^{\prime}$ imply (ii).

Conversely, assume that (i)-(iii) hold. Since $E$ is quasibarrelled it is sufficient to show that $E$ is semi-Montel. To this end, we have to prove that every closed bounded subset $A$ of $E$ is compact. Since $E$ is quasi-complete, it suffices to show that $A$ is precompact, see Theorem 3.4.1 of [34]. Suppose for a contradiction that $A$ is not precompact. Then, by Theorem 5 of [3], there is a neighborhood $U$ of zero and a sequence $S=\left\{a_{n}: n \in \mathbf{N}\right\}$ in $A$ such that

$$
a_{n}-a_{m} \notin U \text { for every } n \neq m \text {. }
$$

Proposition 3.6 and (ii) imply that $E$ does not contain $\ell_{1}$. Therefore, by the Rosenthal property (Proposition 2.11), $S$ contains a weakly Cauchy subsequence $\left\{a_{n_{k}}: k \in \mathbf{N}\right\}$. In particular $a_{n_{k+1}}-a_{n_{k}} \rightarrow 0$ in the weak topology, and hence $a_{n_{k+1}}-a_{n_{k}} \rightarrow 0$ in the topology of $E$ by the Schur property. But this contradicts (3.1). Thus $E$ is semi-Montel.

The next proposition extends (a) of Proposition 11 in [5] and has a similar proof.

Proposition 3.8. Let $E$ be a $c_{0}$-barrelled quasi-complete Mackey space whose every bounded set is metrizable. If $E$ has the Schur property, then also $\left(E^{\prime}, \mu\left(E^{\prime}, E\right)\right)$ has the Schur property. The converse holds if $U^{\circ}$ is $\sigma\left(E^{\prime}, E\right)$-sequentially compact for every neighborhood $U$ of zero in $E$, in particular, if $E$ is separable.

Proof. It follows from Theorem 1.2 and Proposition 2.11 that $E$ weakly respects compactness. Now Proposition 2.12 implies that $\left(E^{\prime}, \mu\left(E^{\prime}, E\right)\right)$ is a subspace of $C_{k}(E, \mathbf{F})$, so $\mu\left(E^{\prime}, E\right)=\left.\tau_{k}\right|_{E^{\prime}}$.

Let $\left\{\chi_{n}: n \in \mathbf{N}\right\}$ be a $\sigma\left(E^{\prime}, E\right)$-null sequence in $E^{\prime}$. Then the $c_{0}$-barrelledness of $E$ implies that the sequence $S=\left\{\chi_{n}: n \in \mathbf{N}\right\} \cup\{0\}$ is equicontinuous. So $S \subseteq U^{\circ}$ for 
some neighborhood $U$ of zero in $E$. Since $\left.\sigma\left(E^{\prime}, E\right)\right|_{U^{\circ}}=\left.\tau_{k}\right|_{U^{\circ}}$ by Proposition 9.3.8 of [28], we obtain $\left.\sigma\left(E^{\prime}, E\right)\right|_{U^{\circ}}=\left.\mu\left(E^{\prime}, E\right)\right|_{U^{\circ}}$. Thus $\chi_{n} \rightarrow 0$ in $\mu\left(E^{\prime}, E\right)$.

Now assume that $U^{\circ}$ is $\sigma\left(E^{\prime}, E\right)$-sequentially compact for every neighborhood $U$ of zero in $E$ and every $\sigma\left(E^{\prime}, E\right)$-convergent sequence in $E^{\prime}$ is $\mu\left(E^{\prime}, E\right)$-convergent. Suppose for a contradiction that $E$ is not a Schur space. So there is a weakly null sequence $S=\left\{x_{n}: n \in \mathbf{N}\right\}$ in $E$ such that $x_{n} \not \rightarrow 0$ in $E$. Hence there exists an absolutely convex closed neighborhood $U$ of zero in $E$ such that $S \backslash U$ is infinite. By passing to a subsequence we can assume that $S \cap U=\emptyset$. For every $n \in \mathbf{N}$ choose $\chi_{n} \in U^{\circ}$ such that $\left|\chi_{n}\left(x_{n}\right)\right|>1$. Since $U^{\circ}$ is $\sigma\left(E^{\prime}, E\right)$-sequentially compact, by passing to a subsequence if needed, we can additionally assume that $\chi_{n}$ weakly converges to some $\chi \in U^{\circ}$. By the Schur property we obtain $\chi_{n} \rightarrow \chi$ in $\mu\left(E^{\prime}, E\right)$. Since the set $K=\overline{\operatorname{acx}}(S)$ is weakly compact by Theorem 5.1.11 of [35], it follows that

$$
A_{n}:=\sup \left\{\left|\left(\chi_{n}-\chi\right)(x)\right|: x \in K\right\} \rightarrow 0 \text { at } n \rightarrow \infty
$$

Therefore

$$
1<\left|\chi_{n}\left(x_{n}\right)\right| \leq\left|\left(\chi_{n}-\chi\right)\left(x_{n}\right)\right|+\left|\chi\left(x_{n}\right)\right| \leq A_{n}+\left|\chi\left(x_{n}\right)\right| \rightarrow 0,
$$

a contradiction.

Acknowledgments. I am deeply indebted to Professor J. Bonet for useful remarks and sending me [5]. I wish to thank the referee for careful reading and useful suggestions.

\section{References}

[1] Albanese, A. A., J. Bonet, and W. J. Ricker: Grothendieck spaces with the DunfordPettis property. - Positivity 14, 2010, 145-164.

[2] Banakh, T., and S. Gabriyelyan: On the $C_{k}$-stable closure of the class of (separable) metrizable spaces. - Monats. Math. 180, 2016, 39-64.

[3] Banakh, T., S. Gabriyelyan, and I. Protasov: On uniformly discrete subsets in uniform spaces and topological groups. - Mat. Stud. 45, 2016, 76-97.

[4] Bierstedt, K. D., and J. Bonet: Density conditions in Fréchet and (DF)-spaces. - Rev. Mat. Complut. 2, 1989, 59-75.

[5] Bonet, J., and M. Lindström: Convergent sequences in duals of Fréchet spaces. - In: Functional Analysis, Proc. of the Essen Conference, Marcel Dekker, New York, 1993, 391-404.

[6] Bonet, J., M. Lindström, and M. Valdivia: Two theorems of Josefson-Nissenzweig type for Fréchet spaces. - Proc. Amer. Math. Soc. 117, 1993, 363-364.

[7] Borwein, J., M. FAbian, and J. VAnderwerfF: Characterizations of Banach spaces via convex and other locally Lipschitz functions. - Acta Math. Vietnam. 22, 1997, 53-69.

[8] Bourgain, J., and H. P. Rosenthal: Martingales valued in certain subspaces of $L^{1}$. - Israel J. Math. 37, 1980, 54-75.

[9] Carrión, H., P. Galondo, and M. L. Lourenço: A stronger Dunford-Pettis property. Studia Math. 184, 2008, 205-216.

[10] Dales, H. G., F. K. Dashiell, JR., A. T.-M. Lau, and D. Strauss: Banach spaces of continuous functions as dual spaces. - Springer, 2016.

[11] Díaz, J. C.: Montel subspaces in the countable projective limits of $L^{p}(\mu)$-spaces. - Canad. Math. Bull. 32, 1989, 169-176.

[12] Diestel, J.: Sequences and series in Banach spaces. - Grad. Texts in Math. 92, Springer, 1984. 
[13] Domanski, P., and L. Drewnowski: Fréchet spaces of continuous vector-valued functions: Complementability in dual Fréchet spaces and injectivity. - Studia Math. 102, 1992, 257-267.

[14] Domínguez, X.: Grupos Abelianos topológicos y sumabilidad. - Doctoral dissertation, Universidad Complutense de Madrid, 2001.

[15] Dowling, P. N., D. Freeman, C. J. Lennard, E. Odell, B. Randrianantoanina, and B. TuretT: A weak Grothendieck compactness principle. - J. Funct. Anal. 263, 2012, 13781381.

[16] Edwards, R. E.: Functional analysis. - Reinhart and Winston, New York, 1965.

[17] Engelking, R.: General topology. - Panstwowe Wydawnictwo Naukowe, 1985.

[18] Fabian, M., P. Habala, P. Hájek, V. Montesinos, J. Pelant, and V. Zizler: Banach space theory. The basis for linear and nonlinear analysis. - Springer, New York, 2010.

[19] Gabriyelyan, S.: Topological properties of the group of the null sequences valued in an Abelian topological group. - Topology Appl. 207, 2016, 136-155.

[20] Gabriyelyan, S.: On the Ascoli property for locally convex spaces. - Topology Appl. 230, 2017, 517-530.

[21] Gabriyelyan, S.: Maximally almost periodic groups and respecting properties. arXiv:1712.05521.

[22] Gabriyelyan, S., J. Ka̧kol, A. Kubzdela, and M. Lopez Pellicer: On topological properties of Fréchet locally convex spaces with the weak topology. - Topology Appl. 192, 2015, 123-137.

[23] Galindo, J., and S. Hernández: The concept of boundedness and the Bohr compactification of a MAP abelian group. - Fund. Math. 159, 1999, 195-218.

[24] Gillman, L., and M. Jerison: Rings of continuous functions. - Van Nostrand, New York, 1960.

[25] Hájek, P., V. Montesinos, J. Vanderwerff, and V. Zizler: Biorthogonal systems in Banach spaces. - Springer, 2008.

[26] Hernández, S., J. Galindo, and S. Macario: A characterization of the Schur property by means of the Bohr topology. - Topology Appl. 97, 1999, 99-108.

[27] Hernández, S., and S. Macario: Invariance of compactness for the Bohr topology. - Topology Appl. 111, 2001, 161-173.

[28] Horváth, J.: Topological vector spaces and distributions, I. - Addison-Wesley, Reading, Mass, 1966.

[29] Jaramillo, J. A., A. Prieto, and I. Zalduendo: Sequential convergences and DunfordPettis properties. - Ann. Acad. Sci. Fenn. Math. 25, 2000, 467-475.

[30] Jarchow, H.: Locally convex spaces. - B. G. Teubner, Stuttgart, 1981.

[31] Johnson, W. B., R. Lillemets, and E. Oja: Representing completely continuous operators through weakly $\infty$-compact operators. - Bull. London Math. Soc. 48, 2016, 452-456.

[32] Köтнe, G.: Topological vector spaces, Vol. I. - Springer-Verlag, Berlin, 1969.

[33] Lindenstrauss, J., and L. Tzafriri: Classical Banach spaces I. - Springer, Berlin, 1977.

[34] Narici, L., and E. Beckenstein: Topological vector spaces. Second edition. - CRC Press, New York, 2011.

[35] Pérez Carreras, P., and J. Bonet: Barrelled locally convex spaces. - North-Holland Math. Studies 131, Amsterdam, 1987.

[36] Pethe, P., and N. Thakare: Note on Dunford-Pettis property and Schur property. - Indiana Univ. Math. J. 27, 1978, 91-92.

[37] Pryce, J. D.: A device of R. J. Whitley's applied to pointwise compactness ' in spaces of continuous functions. - Proc. London Math. Soc. 23, 1971, 532-546. 
[38] Rosenthal, H. P.: A characterization of Banach spaces containing $\ell^{1}$. - Proc. Natl. Acad. Sci. USA 71, 1974, 2411-2413.

[39] Ruess, W.: Locally convex spaces not containing $\ell_{1}$. - Funct. Approx. Comment. Math. 50, 2014, 351-358.

[40] Valdivia, M.: Some new results on weak compactness. - J. Funct. Anal. 24, 1977, 1-10.

[41] Valdivia, M.: On totally reflexive Fréchet spaces. - In: Actti del Convegno Internazionale in Analisi Matematico e sue Applicazioni, Dedicato Prof. G. Aquaro, Bari 1991, 39-55.

[42] Wilansky, A.: Modern methods in topological vector spaces. - McGraw-Hill, 1978.

Received 9 May 2018 • Accepted 6 September 2018 